\title{
Enhancement of two photon fluorescence collection by using effectively single mode double clad hollow core fiber with low dispersion at $800 \mathrm{~nm}$
}

\author{
Hanna Stawska • Elżbieta Bereś-Pawlik
}

Received: 3 March 2014 / Accepted: 3 July 2014 / Published online: 17 July 2014

(C) The Author(s) 2014. This article is published with open access at Springerlink.com

\begin{abstract}
We show that application of the double clad hollow core fiber (DCHCF) significantly affects efficiency of collecting a two photon fluorescence signal. In our approach we propose a new construction of the hollow core double clad fiber which can be used to send simultaneously an ultrafast signal through the single-mode air core and collect the florescence signal through a multimodal clad. The presented fiber has a dispersion equal to $5.13 \mathrm{ps} / \mathrm{nm} \mathrm{km}$ and losses $0.5 \mathrm{~dB} / \mathrm{m}$ in the range of $800 \mathrm{~nm}$. In the wavelengths range between 770 to $850 \mathrm{~nm}$ the fiber is endlessly single mode with higher order modes HOM's losses above $17 \mathrm{~dB} / \mathrm{m}$. The use of such a fiber allows elimination of prevalent precompensation dispersion systems and considerably simplifies the two photon fluorescence endoscopy setup.
\end{abstract}

Keywords Photonic crystal fiber - Two-photon endoscopy · Fluorescence endoscopy · Ultrafast signals

\section{Introduction}

Two photon fluorescence is a phenomenon which provides a new quality for noninvasive imaging of biological tissue. It allows to obtain higher resolution in comparison to one photon fluorescence and it has a better ability to penetrate the tissue due to the fact that in two photon fluorescence infrared light is applied (Elahi and Wang 2011; Yicong, Xingde 2010). Moreover, the risk of tissue's damage caused by the light is lower. The most commonly used endoscopic setup design involves the use of a double clad fiber. In this case, the core of the fiber is used for transmission of the excitation signal, whereby the inner clad is used for receiving fluorescent signal. But delivery of ultra-short pulses with a flexible optical fiber is very difficult to achieve due to the fact that femtosecond pulses suffer from temporal and spectral broadening especially operating near $800 \mathrm{~nm}$ wavelength, where normal dispersion

H. Stawska $(\bowtie) \cdot$ E. Bereś-Pawlik

Telecommunications and Teleinformatics Department, Wroclaw University of Technology,

Wybrzeże Wyspiańskiego 27, 50-370 Wrocław, Poland

e-mail: hanna.stawska@pwr.wroc.pl 


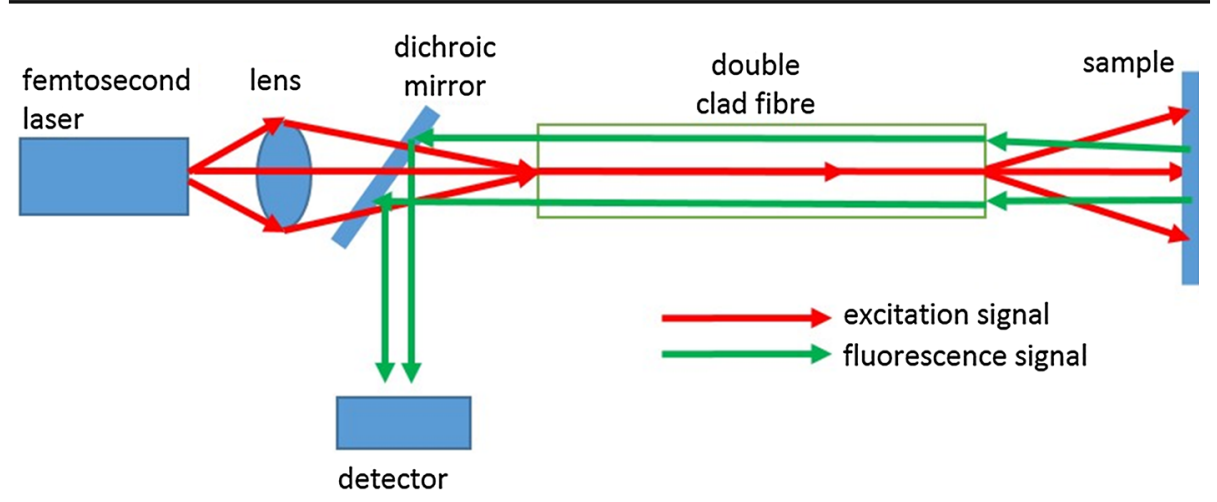

Fig. 1 Simplified endoscopic setup used to determine enhancement of fluorescence collection

is large (Clark et al. 2001; Kalashyan et al. 2012). Therefore, in order to design an efficient setup for two-photon endoscopy it is necessary to compensate dispersion.

In our prior work Stawska, Bereś-Pawlik (2012) we showed that it is possible to design double clad fibers with very small dispersion at $800 \mathrm{~nm}$. We analyzed two constructions: one with Ge-doped rods and the other with a hollow core. During our simulation it occurred that dispersion of the Ge-doped fiber is strongly nonlinear and little change of wavelength causes a big change of dispersion. In the case of a hollow core the dispersion slope is relatively low but the dispersion is still too large to use in propagating ultrafast signals without specialized dispersion compensation systems.

In this approach a new structure of a double clad hollow core is presented. The numerical simulation shows that this fiber has a very low loss and dispersion in the range of $809 \mathrm{~nm}$. We also show that application of such a fiber can significantly improve two photon fluorescence collection efficiency and simplify the two-photon endoscopy setup by elimination of the dispersion compensation systems.

The sections of this paper are as follows: in Sect. 2 we show derivation of the formula for the time-averaged photon fluorescence flux which is needed for determination of enhancement of two photon fluorescence collection efficiency. In Sect. 3, we present the new structure of a double clad hollow core fiber and calculated losses and dispersion for its core modes. In Sect. 4, we outline the method used to determine the enhancement of two-photon collection efficiency and we present numerical results.

\section{Theoretical consideration}

For our consideration we assumed a simplified endoscopic setup consisting of a femtosecond laser, dichroic mirror, lens, fiber detector and sample (Fig. 1). We also assume that the setup will be the same regardless of which fiber will be used, so further considerations ignore the impact of other elements on the efficiency of the signal.

Thus, in order to determine the enhancement of collection efficiency by using a hollow core double clad fiber we have to determine the number of fluorescence photons $N_{f l}$ collected by this fiber and by a standard double clad fiber. Thus we assume that the number of fluorescence photons $N_{f l}$ collected per unit time is proportional to fluorescence quantum efficiency $\eta$, fluorescence collection efficiency $\rho$, laser to fiber coupling efficiency $D$, number of photons absorbed per the unit of time $N_{a b s}$. On the other hand we know that the total number of photons 
absorbed by means of two photon endoscopy (TPE) is a function of two-photon absorption (TPA) cross section, square of the incident intensity $I$, illuminated sample volume $V$ and dye concentration $C$ (Xu and Webb 1996). Thus we can write

$$
N_{f l}(t)=C D \sigma \rho \eta \int_{V} I^{2}(r, t) d V
$$

TPA cross section $\sigma$ denotes the efficiency of a particular molecule in the ground state to reach the excited state via a TPA process.

$$
\sigma=\frac{\hbar \omega \beta}{N_{A} \rho_{c} \times 10^{-3}}
$$

where $\hbar$ is reduced Planck constant, $N_{A}$ is Avogadro's number, and $\rho_{c}$ is the concentration in mole per liter (Ajami et al. 2010). Assuming no dependence between special and temporal distribution of light $I(r, t)=S(r) I(t)$ where, $S(r)$ is unitless special distribution function, we can rewrite Eq. (1) in the following form:

$$
N_{f l}(t)=C D \sigma \rho \eta I^{2}(t) \int_{V} S^{2}(r) d V
$$

Due to the fact that we measure time-averaged photon fluorescence flux we obtain (Xu and Webb 1996)

$$
\left\langle N_{f l}(t)\right\rangle=C D \sigma \rho \eta g\langle I(t)\rangle^{2} \int_{V} S^{2}(r) d V
$$

where $g=<I^{2}(t)>/<I(t)>^{2}$ is measure of the second-order temporal coherence of the excited source Loudon (1983).

To simplify the calculation we approximate a Gaussian profile of the excitation beam with a disc of radius $w(z)$ with a constant intensity $I$. Applying the law of conservation of energy and assuming Gaussian beam we can obtain dependence between instantaneous intensity and power:

$$
I(t)=\frac{2 P(t)}{\pi w(z)^{2}}
$$

where $w(z)$ is laser beam radius at position $z$. The peak intensity for Gaussian beam is two times higher than it is often assumed and this formula can be verified by integrating the intensity over the whole beam area (Quimby 2006).

Additionally, assuming that temporal coherence of the exciting source depends only on the duty cycle and the shape of the pulse we can write:

$$
\left\langle N_{f l}(t)\right\rangle=C D \sigma \rho \eta \frac{2^{*} 0.664}{\tau R}\left\langle\frac{P(t)}{\pi w(z)^{2}}\right\rangle_{V}^{2} \int_{V} S^{2}(r) d V
$$

In our configuration (no lenses at the distal tip of fiber) the probe volume is determined by excitation profile of the laser beam. Thus we rewrite Eq. (6) as:

$$
\left\langle N_{f l}(t)\right\rangle=C D \sigma \rho \eta \frac{2^{*} 0.664}{\tau R \pi w(z)^{2}}\langle P(t)\rangle^{2}
$$

where $\tau$ is the output pulse width and $\mathrm{R}$ is the laser repetition rate. 


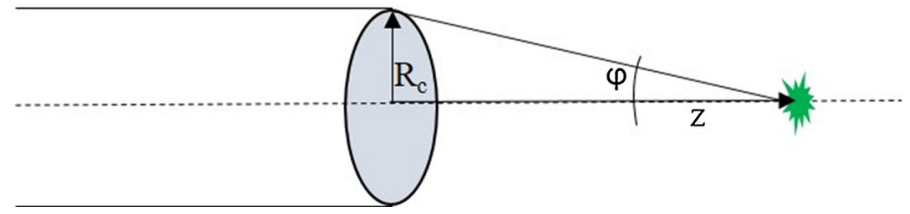

Fig. 2 Determining angle of ray $\varphi$ hitting the fiber which allows the incident light to be guided

Therefore to determine enhancement of fluorescence collection we have to designate the width of output pulse and the fluorescence collection efficiency.

\subsection{Determining output pulse width}

In order to simplify estimation of pulse broadening we assume operation at low power. Thus we can neglect nonlinear effects which can appear during propagation of femtosecond signals through the fiber. In the case of a single mode fiber where there is no modal dispersion and for Gaussian pulse, dispersion causes the output pulse width to be Agrawal (2007):

$$
\tau=\tau_{i n}\left[1+\left(\frac{L}{L_{D}}\right)^{2}\right]^{1 / 2}
$$

where $L_{D}=\tau_{i n}^{2} /\left|\beta_{2}\right|$ is dispersion length and $\beta_{2}$ is group velocity dispersion.

\subsection{Determining collection efficiency of fiber}

Assuming that the fluorescence signal is emitted in all directions we can infer that collecting efficiency will be proportional to the solid angle $\Omega$ subtended by the angle $\varphi$ (Fig. 2). Thus we can write $\rho=\Omega / 4 \pi$.

On the other hand we know that the maximum angle of a ray $\varphi_{\max }$ (against the fiber axis) hitting the fiber core which allows the incident light to be guided by the core is defined as an acceptance angle and is defined as:

$$
N A=\sin \varphi_{\max }
$$

Thus knowing that $\Omega=2 \pi(1-\cos \varphi)$ we can obtain the following expression for fluorescence efficiency depending on the position of the source of fluorescence (samples) from the face of the fiber.

$$
\rho(z)= \begin{cases}\frac{1}{2}\left(1-\sqrt{1-N A^{2}}\right), & \text { for } z \leq \frac{R_{c}}{N A} \sqrt{1-N A^{2}} \\ \frac{1}{2}\left(1-\frac{z}{\sqrt{z^{2}+R_{c}^{2}}}\right), & \text { for } z>\frac{R_{c}}{N A} \sqrt{1-N A^{2}}\end{cases}
$$

In the case of double clad fibers we assume that for the fluorescence signal inner clad of fiber is working as a core. In order to calculate collecting efficiency of this inner clad we should substitute numerical aperture of this clad to Eq. (10). Obviously, the core of this fiber can also guide the fluorescence signal and then in order to determine collection efficiency of the core we should use NA of the core. 


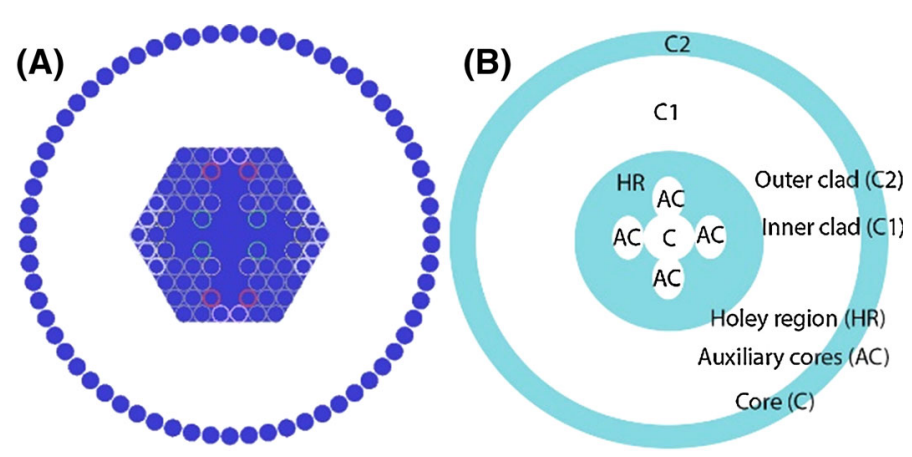

Fig. 3 a Cross section of the examined fiber. Different colors of rings highlight their different thicknesses. Rings are made from silica and filled with air (blue color). b Schematic presentation of the structure. (Color figure online)

\section{Double clad hollow core fiber structure and paramerers}

In order to determine the fluorescence collection efficiency we have to obtain necessary parameters of the proposed double clad hollow core fiber. These parameters are numerical aperture (NA) of the inner clad and the core and dispersion. In our consideration we neglected the losses of the fibers to simplify the calculations.

\subsection{The structure}

Figure 3 shows a hollow core double clad photonic crystal fiber structure that is used in this approach. The background material is silica, and the holes are filled with air (blue color). The holey region consists of silica rings arranged in hexagonal lattice. Different colors of rings highlight their different thicknesses. The main core is formed by removing 7 inner cells and its radius is equal to $\mathrm{R}=10 \mu \mathrm{m}$. Additionally, the structure contains four auxiliary cores which are connected to the main core. The aim of these cores and different thicknesses of the rings is to suppress higher orders modes. The lattice constant $\Lambda=8.1 \mu \mathrm{m}$, the outer radius of rings is equal to $r_{1}=4.05 \mu \mathrm{m}$, the inner diameter of rings $2 \mu \mathrm{m}<\mathrm{r}_{2}<3.3 \mu \mathrm{m}$. In the Fig. $3 \mathrm{~b}$ is shown schematic presentation of the structure of the fiber which comprises the following regions: outer clad (C2), inner clad (C1), holey region (HR), auxiliary cores (AC) and core $(\mathrm{C})$.

\subsection{Losses and dispersion of double clad hollow core fiber (DCHCF)}

In this approach commercially available application Lumerical Mode Solution was used to solve the Maxwell equations and to obtain the effective index $n_{\text {eff }}$ and losses $\alpha$ of this fiber. Lumerical Mode Solution is widely used to accurately calculate the physical properties of guided modes in different arbitrary waveguides: photonic crystal fibers, rib waveguides, surface plasmon waveguides etc (www.lumerical.com). It was used, among others, to calculate group velocity dispersion of the fundamental mode of a microstructured fiber in $\mathrm{Hu}$ et al. (2012) and to calculate the effective refractive index and losses of the fundamental mode of the hollow core fiber in Boucon et al. (2008). Moreover this application is cited by more than 800 publications (www.lumerical.com).

In order to solve Maxwell equations Lumerical Mode Solution uses finite difference time domain method. It also uses rectangular mesh and electric and magnetic fields are 

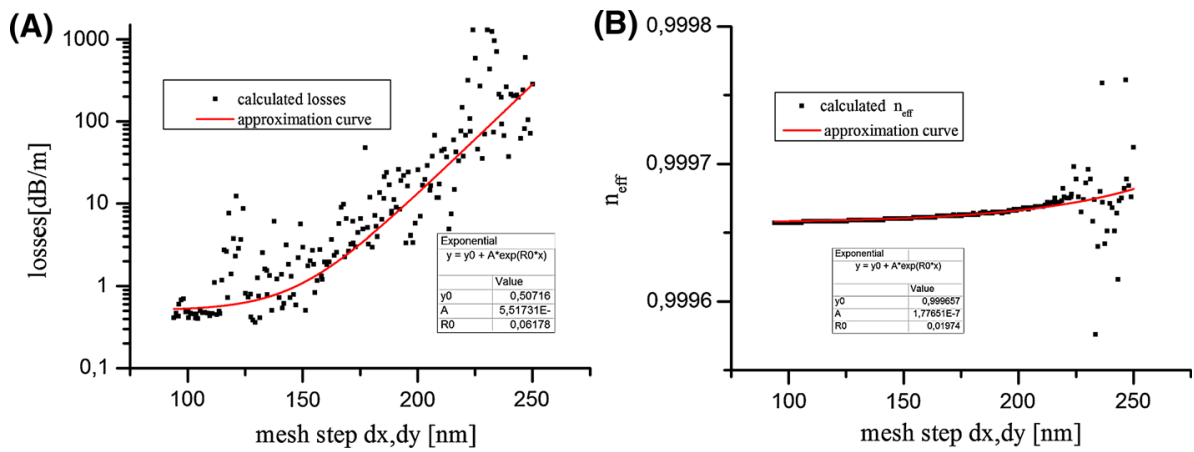

Fig. 4 Calculated losses $\mathbf{a}$ and effective refractive index $\mathbf{b}$ of the fundamental core mode in the mesh size range of $90-250 \mathrm{~nm}$

calculated at each mesh point (www.lumerical.com). Therefore it is important to determine the right mesh size. It is obvious that the smaller mesh step the more accurate calculations but this is connected with increasing simulation time and memory requirements. So to test the convergence we vary mesh size $\mathrm{dx}$ and dy in dozens steps and we check if the results stop changing. We perform the convergence test for $800 \mathrm{~nm}$ wavelength. In the Fig. 4 are shown calculated losses A) and effective refractive index B) for the fundamental mode for different mesh sizes from the range of 90-250 nm. Optimally, we should reduce dx and dy until the results (losses and $\mathrm{n}_{\mathrm{eff}}$ ) stop changing but in our case we cannot decrease mesh size below $90 \mathrm{~nm}$ because we obtain factorization matrix error. To illustrate convergence appropriately, we approximate calculated points with exponential curves. As one can observe, the limit at zero is about $0.5 \mathrm{~dB} / \mathrm{m}$ for losses and 0.999657 for the $\mathrm{n}_{\text {eff }}$.

Additionally to better present the idea of testing convergence we also define at each step of simulation the difference with the result of a previous step for losses and for effective index as follow:

$$
\begin{aligned}
\Delta \alpha & =\left|\alpha_{i+1}-\alpha_{i}\right| \\
\Delta n_{e f f} & =\left|n_{e f f_{i+1}}-n_{e f f_{i}}\right|
\end{aligned}
$$

where $\mathrm{i}$ is the step for mesh size.

As it can be noticed from the Fig. 4a when the mesh size is lower than $120 \mathrm{~nm}$ value of losses is almost constant and the difference between the successive results for losses $\Delta \alpha$ is smaller than 0.2 (Fig. 5a). We did not obtain the value zero as in the case of the $\Delta$ neff. (Fig. 5b). In the case of $\Delta \alpha$ we assume that the nonzero value can be caused by different source of error than too big mesh size for example refraction from PML layers or stair-case error [11]. For our consideration we assume that the $\Delta \alpha<0.2$ is on the acceptable level and for further calculations we assume $\mathrm{dx}=100 \mathrm{~nm}$ and $\mathrm{dy}=100 \mathrm{~nm}$

It is also worth mentioning that our calculations have been carried out in Wroclaw Centre for Networking and Supercomputing and were performed on the Supernova cluster with more than $10.4 \mathrm{~TB}$ of available memory. In order to determine the dispersion we defined 200 tasks in the range of 750 to $850 \mathrm{~nm}$ with step $0.5 \mathrm{~nm}$. For each task we reserved 10GB of the memory.

Knowing dependence $\mathrm{n}_{\text {eff }}$ on $\lambda$ it is very simple to obtain group velocity dispersion (GVD). The structure of the fiber was selected to provide the least attenuation and GVD in the vicinity of the $800 \mathrm{~nm}$. In the Fig. 6 are shown calculated losses A) and dispersion B) in the wavelength 

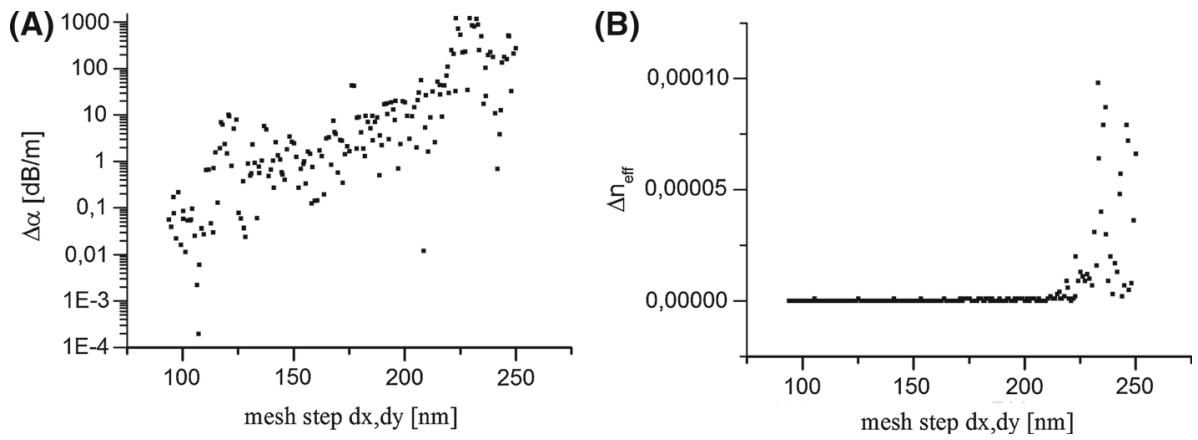

Fig. 5 Difference between the current and the previous step for $\mathbf{a}$ losses and for $\mathbf{b}$ effective index $\mathbf{a}$
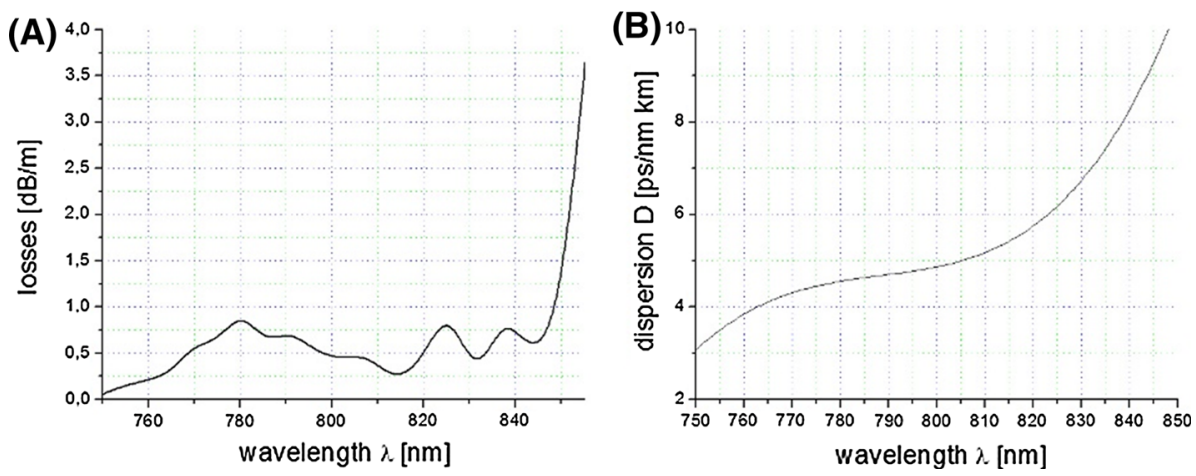

Fig. 6 Calculated losses $\mathbf{a}$ and dispersion $\mathbf{b}$ of the fundamental core mode in the wavelength range of 750$850 \mathrm{~nm}$.

range from 750 to $850 \mathrm{~nm}$ for the fundamental core mode. As we mentioned previously, the higher order modes are suppressed due to applying four auxiliary cores and HOMs have losses $>17 \mathrm{~dB} / \mathrm{m}$ at the range of 770 to $850 \mathrm{~nm}$.

The calculated dispersion at $809 \mathrm{~nm}$ is equal to $5.13 \mathrm{ps} / \mathrm{nm} \mathrm{km}$. It is about 20 times smaller than in fused silica and has the opposite sign so it is very easy to compensate $(5 \mathrm{~cm}$ of bulk silica can compensate dispersion of $1 \mathrm{~m}$ fiber).

Additionally the collection area of this fiber is not only limited to the area of the second clad but also to the area of a core.

\subsection{Numerical aperture of the inner clad}

In order to calculate numerical aperture of the inner clad $\mathrm{C} 1$ of DCHCF we have to determine effective index of the outer clad C2 (see Fig. 3) with high air-filling fraction. Thus we consider $\mathrm{C} 2$ as a network of the silica slabs (Fig. 7).

We find the effective reflective index of that structure from waveguide slab equations (Wadsworth et al. 2004; Okamoto 2006):

$$
\begin{aligned}
& u \tan (u)=w \\
& u=k a \sqrt{n_{s i}^{2}-\left(\frac{\beta}{k}\right)^{2}}
\end{aligned}
$$


Fig. 7 Cross section of the slab

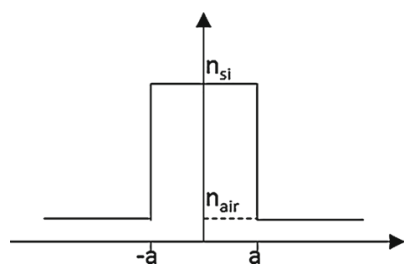

$$
w=k a \sqrt{\left(\frac{\beta}{k}\right)^{2}-n_{a i r}^{2}}
$$

where $a$ is half of the slab's width. Knowing that $n_{\text {eff }}=\frac{\beta}{k}$ and $N A=\sqrt{n_{1}^{2}-n_{\text {eff }}^{2}}$ we can determine numerical aperture of the clad of DCHCF to be $N A_{\text {clad }}=0.82$.

\subsection{Numerical aperture of the core}

In order to determine NA of the core of this fiber we numerically calculated the bandgap's upper edge and using a methodology which is presented in Digonnet et al. (2005) we obtain $N A_{\text {core }}$ from the following formula:

$$
N A_{\text {core }}=\sqrt{1-\left(\frac{k_{u}}{k_{0}}\right)^{2}}=0.055
$$

where $k_{u}$ is bandgap's upper edge and $k_{0}=\omega / c$.

\section{Numerical results}

To determine the enhancement of two-photon fluorescence collection of a double clad hollow core fiber we have to compare collection efficiency of commercially available fibers with collection efficiency of the fiber proposed in this approach. We selected two commercially available fibers: Passive 6/125DC-PM from Liekki (6/125 $\mu \mathrm{m}$ and 0.15/0.46 NA) and DC165-16 Passive from CrystalFiber (16/165 $\mu \mathrm{m}$ and 0.04/0.6 NA) and we used them in our simulations. We assume that these fibers have dispersion $100 \mathrm{ps} / \mathrm{nm} \mathrm{km}$ and the pulse width is $100 \mathrm{fs}$.

In our consideration we assume that collection area of our fiber is the sum of area of inner clad C1 and area of the core C (Fig. 3.) As it can be seen in the Fig. 8. in our fiber the light is propagated through the inner clad $\mathrm{C} 1$ and through the inner core $\mathrm{C}$.

For physical reasons we also assumed that the distance between the fiber and the sample is larger than $300 \mu \mathrm{m}$ (we would like to avoid the situation when the face of the fiber is in contact with the sample). Substituting Eqs. (10) to Eq. (7) we can obtain dependence of collection efficiency of the fiber from the wavelength. We also define enhancement of the collection efficiency of DCHCF as $E=\frac{\left\langle N_{f l}(t)\right\rangle_{D C H C F}}{\left\langle N_{f l}(t)\right\rangle_{S F}}$ where $\left\langle N_{f l}(t)\right\rangle_{D C H C F}$ is the number of fluorescence photons collected by the DCHCF and $\left\langle N_{f l}(t)\right\rangle_{S F}$ is the number of fluorescence photons collected by the standard fiber.

Assuming that our fiber has the core radius $R_{c}=10 \mu \mathrm{m}$, the holey region radius $R_{h}=$ $43 \mu \mathrm{m}$ and the inner clad radius $\mathrm{R}_{\mathrm{C} 1}=85 \mu \mathrm{m}$. The calculated enhancement of collection efficiency of the hollow core double clad fiber is presented in Fig. 9. 

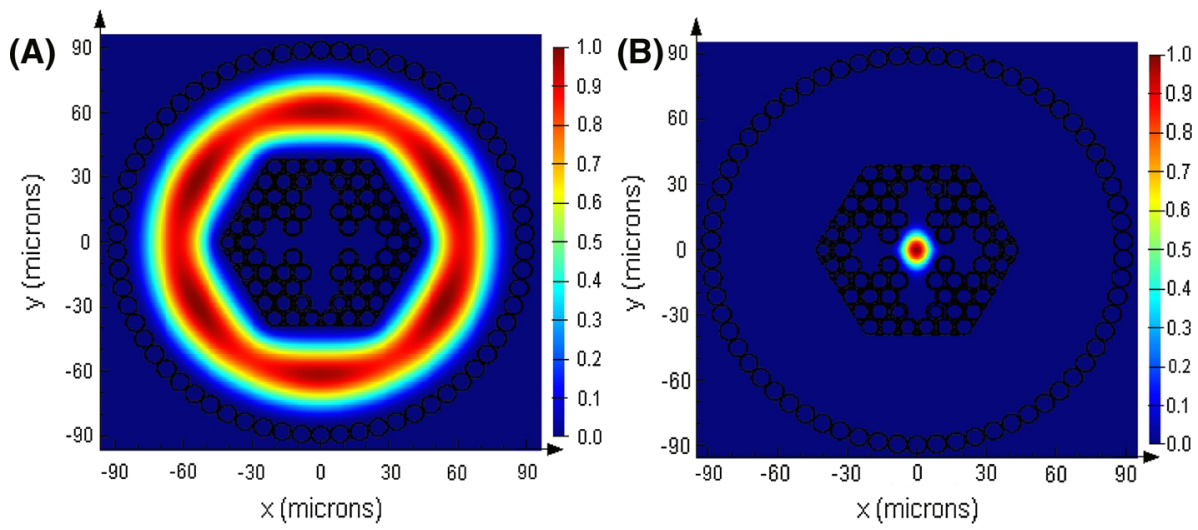

Fig. 8 Near field electric field intensity of the fundamental mode which is propagated in the inner clad C1 a and in the inner core $\mathbf{c}$ at the wavelength $510 \mathrm{~nm}$

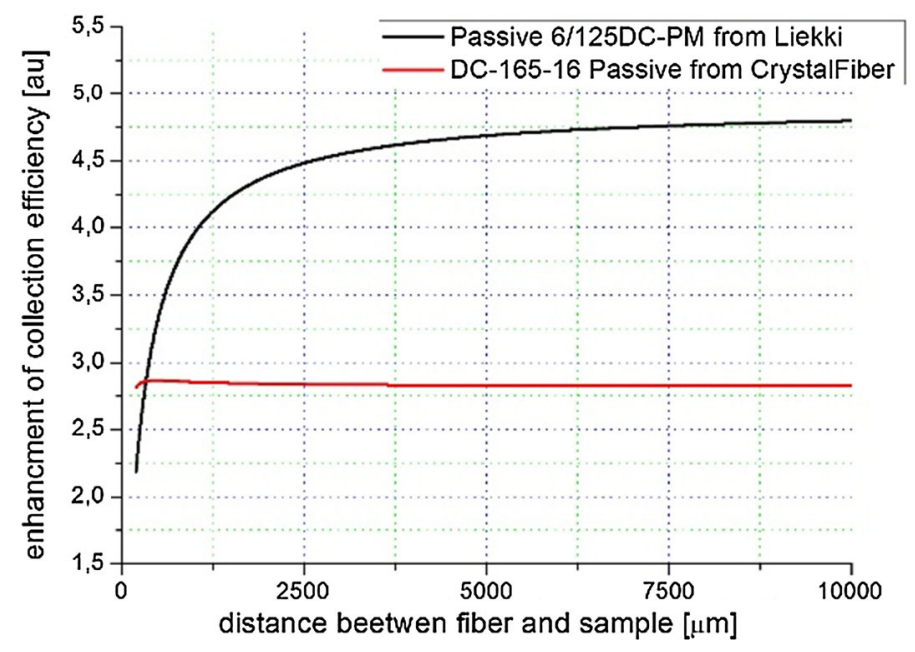

Fig. 9 Enhancement of collection efficiency of double clad hollow core fiber

As it is shown in Fig. 9 the enhancement of collection efficiency by using of DCHCF is significant. In the case of the fiber from the CrystalFiber the enhancement of the collection efficiency is almost constant and tends to 2.75. For the fiber from Liekki efficiency of the fluorescence collection tends to 4.75 .

\section{Conclusion}

The new construction of the endlessly single mode double clad hollow core fiber was presented. This fiber has very low dispersion about $5 \mathrm{ps} / \mathrm{nm} \mathrm{km}$ in the range of $800 \mathrm{~nm}$. Additionally this fiber has very low losses approximately $0.5 \mathrm{~dB} / \mathrm{m}$.

Moreover, the core of the fiber could be used not only to transmit the excitation signal but also to transmit the collection signal. 
We have also demonstrated numerically that application of this fiber significantly improves collection efficiency. At the distance of $10 \mathrm{~mm}$ of the sample from the face of the fiber the value of minimum enhancement of collection efficiency is 3 times bigger than for commercially available fibers.

Acknowledgments This work is co-financed by Grant No. S30011. Calculations have been carried out in Wroclaw Centre for Networking and Supercomputing (http://www.wcss.wroc.pl), Grant No. 184.

Open Access This article is distributed under the terms of the Creative Commons Attribution License which permits any use, distribution, and reproduction in any medium, provided the original author(s) and the source are credited.

\section{References}

Agrawal, G.P.: Nonlinear Fiber Optics, 4th edn. Academic Press, Boston (2007)

Ajami, A., Husinsky, W., Liska, R., Pucher, N.: Two-photon absorption cross section measurements of various two-photon initiators for ultrashort laser radiation applying the Z-scan technique. J. Opt. Soc. Am. B 27, 2290-2297 (2010)

Boucon, A., Alasia, D., Beugnot, J.-C., Mélin, G., Lempereur, S., Fleureau, A., Maillotte, H., Dudley, J.-M., Sylvestre, T.: Supercontinuum generation from 1.35 to $1.7 \mu \mathrm{m}$ by nanosecond pumping near the second zero- dispersion wavelength of a microstructured fiber. Photon. Technol. Lett. IEEE 20(10), 842-844 (2008)

Clark, S., Ilday, F., Wise, F.: Fiber delivery of femtosecond pulses from a Ti:sapphire laser. Opt. Lett. 26, 1320-1322 (2001)

Digonnet, M., Kim, H., Kino, G., Fan, S.: Understanding air-core photonic-bandgap fibers: analogy to conventional fibers. J. Lightw. Technol. 23, 4169-4177 (2005)

Elahi, S.F., Wang, T.D.: Future and advances in endoscopy. J. Biomed. Opt. 4, 471-481 (2011)

HU, D.J.J., Yixin, W., Jun Long, L., Taishi, Z., Milenko, K.B., Zhihao, C., Meng, J., Guanghui, W., Feng, L., Shum, P.P., Qizhen, S., Huifeng, W., Weijun, T., Wolinski, T.R.: Novel miniaturized fabry-perot refractometer based on a simplified hollow-core fiber with a hollow silica sphere tip. Sensors J. IEEE 12(5), $1239-1245$ (2012)

Kalashyan, M., Lefort, C., Martínez-León, L., Mansuryan, T., Mouradian, L., Louradour, F.: Ultrashort pulse fiber delivery with optimized dispersion control by reflection grisms at $800 \mathrm{~nm}$. Opt. Express 20, 2562425635 (2012)

Loudon, R.: The Quantum Theory of Light, 2nd edn, pp. 82-119. Clarendon, Oxford (1983)

Lumerical Mode Solution, www.lumerical.com

Okamoto, K.: Fundamentals of Optical Waveguides, 2nd edn. Academic Press, London (2006)

Quimby, R.S.: Photonics and Lasers: An Introduction, pp. 307-312. Wiley, New York (2006)

Stawska, H., Bereś-Pawlik, E.: Construction of double cladding small dispersion photonic crystal fiber to guide ultrashort pulse at $800 \mathrm{~nm}$. Acta Phys. Polonica. A., vol. 122, nr 5, s. 896-899 (2012)

Wadsworth, W.J., Percival, R.M., Bouwmans, G., Knight, J.C., Birks, T.A., Hedley, T.D., Russell, P.J.: Very high numerical aperture fibers. IEEE Photon. Technol. Lett. 16, 843-845 (2004)

$\mathrm{Xu}, \mathrm{C}$., Webb, W.: Measurement of two-photon excitation cross sections of molecular fluorophores with data from 690 to $1050 \mathrm{~nm}$. J. Opt. Soc. Am. B 13, 481-491 (1996)

Yicong, W., Xingde, L.: Two-photon Fluorescence Endomicroscopy. Advances in Lasers and Electro Optics. In Costa N and Cartaxo A (ed.), ISBN: 978-953-307-088-9. InTech (2010) 